\title{
Recurrence of Mycosis Fungoides on Multiple Melanocytic Nevi: A Case Report and Review of the Literature
}

\author{
Valeria Brazzelli $^{\text {a }}$ Vincenzo Grasso $^{a} \quad$ Nicolò Rivetti $^{a}$ \\ Giacomo Fiandrino $^{b}$ Marco Lucioni $^{\text {b }}$ Giovanni Borroni $^{\mathrm{a}}$ \\ ${ }^{a}$ Department of Pediatric Sciences and Human and Hereditary Pathology, \\ Institute of Dermatology, and ${ }^{\mathrm{b}}$ Department of Pathology, University of Pavia \\ and Foundation IRCCS Policlinico San Matteo, Pavia, Italy
}

\section{Key Words}

Mycosis fungoides · Melanocytic nevi $\cdot$ Halo phenomenon $\cdot$ PUVA therapy

\begin{abstract}
Melanocytic nevi represent a widespread cutaneous finding. Nevertheless, the presence of mycosis fungoides and melanocytic nevi in the same location is an extremely rare event. We report the case of a patient affected by mycosis fungoides and treated with PUVA therapy, with complete remission of the disease. Eight years after therapy discontinuation, he presented epidermal scaling and an erythematous perinevic halo on 3 old melanocytic lesions, the clinical aspect of which was highly suggestive for Meyerson nevi. The histological and immunohistochemical examination of an excised melanocytic lesion revealed histological features consistent with the diagnosis of mycosis fungoides superimposed on junctional melanocytic nevi. The finding of patches of mycosis fungoides superimposed on melanocytic nevi is a rare event; the confounding clinical appearance with eczematous changes around a pre-existing nevus may recall the halo dermatitis known as Meyerson phenomenon; this highlights the importance of clinical and histological examination to make the correct diagnosis of dermatological diseases.
\end{abstract}

\section{Introduction}

Mycosis fungoides (MF) is the most common type of cutaneous T-cell lymphoma (CTCL) and is characterized by malignant proliferation of skin-homing CD4+ T cells of 
unknown etiology. Clinically, eczematous and/or psoriasiform patches and plaques are the most common presentation, which may progress to cutaneous tumors or involvement of lymph nodes and visceral organs [1].

Despite representing over two thirds of cutaneous lymphoma, MF is not a common disease and its incidence has been estimated to $0.36 / 10^{5}$ person-years in the USA and $0.5 / 10^{5}$ in the Western world [2]. Furthermore, MF has been described in patients with other hematological disorders or malignant solid tumors, and patients with MF have a higher frequency (6-16\%) of second (non-hematological) malignancies, such as melanoma, than the general population [3]. MF has also been described in the context of non-melanoma skin carcinomas or as collision tumors with common or dysplastic melanocytic nevi, even if it is a rare event $[4,5]$.

Meyerson nevus is a melanocytic nevus with an associated acute inflammatory response, producing a peripheral ring of erythema and scale (Meyerson phenomenon). We report the case of a patient with MF treated with PUVA therapy who experienced a disease relapse only on pre-existing melanocytic nevi, with the clinical features mimicking Meyerson phenomenon.

\section{Case Report}

A 63-year-old man presented with multiple, slightly pruritic, erythematous-scaling patches with a cigarette paper-like appearance on his trunk, buttocks, and lower limbs, characterized by a relapsing clinical course over the last 5 months and resistance to common topical therapies.

Histological examination of a cutaneous biopsy of the trunk revealed a band-like lymphocytic infiltrate at the dermoepidermal junction, with a perivascular localization in the superficial dermis and diffuse interstitial infiltrates in the papillary dermis. The presence of lymphocytic exocytosis with nuclear atypia and prominent intraepidermal lymphocytes and the absence of spongiosis were documented. Epidermal parakeratosis was evident.

Immunohistochemistry showed a CD2+, CD3+ and CD4+ lymphocytic infiltrate, with low CD7 expression, while polymerase chain reaction (PCR) analysis revealed a clonal rearrangement of T-cell receptor- $\gamma$ (TCR- $\gamma$ ). A diagnosis of MF was made. The patient presented with patch disease involving $>10 \%$ of the body surface area, without lymph node and visceral involvement. In consideration of these findings, a diagnosis of an early-stage MF (IB) was rendered [6].

The execution of an accurate hematological, biochemical, and imaging screening allowed the exclusion of extracutaneous involvement. According to EORTC guidelines [6], the patient was therefore treated with PUVA therapy, and a clinical remission was documented after 30 treatments, corresponding to a cumulative dose of $195 \mathrm{~J} / \mathrm{cm}^{2}$.

The follow-up showed a lasting remission of the disease over the next 8 years. Yet, the last physical examination evidenced the presence of epidermal scaling and an erythematous perinevic halo on 3 long-standing melanocytic lesions on his trunk; the clinical appearance of the lesions was highly suggestive of Meyerson nevi (fig. 1).

One of the lesions was surgically removed for histological examination with complete elliptical excision followed by primary suture. The nevus was mostly composed of melanocytic nests at the dermoepidermal junction, associated with particularly abundant band-like lymphocytic infiltrates obscuring the dermoepidermal junction, and characterized by focal epidermotropism of hyperchromatic, enlarged, convoluted lymphocytes (i.g. 2a-c). Immunohistochemistry confirmed S100 protein-positive staining in melanocytic nevus cells (fig. $2 \mathrm{~d}$ ), while the infiltrate was predominantly composed of CD3+, CD4+ and CD5+ T cells (fig. 2e, f), with low CD7 expression. These findings were consistent with the diagnosis of MF superimposed on a junctional melanocytic nevus. 
The other two lesions were therefore surgically removed in the same way as described for the previous lesion, and all specimens revealed the same histological features, confirming the diagnosis of MF associated with junctional melanocytic nevi. No further treatment was rendered except surgical excision.

The patient is still in fairly good clinical condition without MF cutaneous lesions and the remission has so far lasted 2 years.

\section{Discussion}

Mycosis fungoides represents the most common type of CTCL, comprising $50 \%$ of CTCLs with a yearly incidence of 0.36 cases per 100,000 of the population; which has remained constant over the past decade, thus being a rare disease [7]. On the contrary, melanocytic nevi represent a widespread cutaneous finding. Nevertheless, the finding of MF and melanocytic nevi in the same location is a rare event; to our knowledge, only 2 cases have previously been described in the literature [5]. In the case we report, new MF lesions relapsed on pre-existing junctional nevi after a PUVA-induced long-lasting remission.

As described by Boyd and Rapini [8], melanocytic lesions appear to be the most common component of cutaneous collision tumors, followed by basal-cell carcinomas and seborrheic keratoses.

McNiff and Glusac [5] reported the first 2 descriptions of a collision between MF and melanocytic neoplasms. In the first case, the occurrence of MF in a congenital nevus was associated with a halo phenomenon restricted to the affected region, thus raising questions about the role of CD8+ cytotoxic T cells in both processes (halo phenomenon and juvenile MF); in the other patient, nests of two morphologies (lymphocytic and melanocytic) in the same specimen presented an unusual histological picture with a confounding and possibly pseudomalignant appearance [5].

In our case, the clinical differential diagnosis of MF superimposed on melanocytic lesions includes, firstly, Meyerson phenomenon. In particular, this condition refers to a well-documented inflammatory reaction described in a variety of cutaneous lesions, including the original description in nevocellular nevi [9]. Although there are many hypotheses for the pathogenesis of Meyerson phenomenon, its etiology remains unclear, and it is still a rare event, characterized by a benign and non-recurring course [10].

On the other hand, mycosis fungoides represents a cancer of $\mathrm{T}$ cells whose behavior is dominated by their propensity to go to the skin, to be activated and persist in an activated state, and then achieving their peculiar clonal dominance [11].

PUVA treatment for MF acts by stimulating CD4+ T cells to produce Th1 cytokines IFN- $\gamma$ and IL-2, which activate tumor-specific CD8+ cytotoxic T lymphocytes (CTL). PUVA-treated CD4+ T cells contemporarily activate monocytes to produce cytokines, such as IL-8, and to become tumor antigen-presenting cells for the development of CD8+ CTL, which finally lyse CTCL cells by overcoming suppression caused by Th2 cytokines released from CTCL cells $[12,13]$. These complex mechanisms suggest that PUVA treatment is not merely cytotoxic, but induces activational events in different cell types, and melanocytes are targeted too. 
It has been demonstrated that UV-induced molecular effects on melanocytes reflect cellular responses that might cause signal transduction and activation of intracellular stress response mechanisms, thus controlling cell communication and immune responses $[14,15]$.

These observations may suggest a trigger role of UVA in eliciting a particular response driven by melanocytes and resulting in a delayed relapse of the disease; at the same time, the collision of these processes may likely represent a casual finding, because our patient presented a localized relapse of MF, not involving the majority of melanocytic nevi, and at the time of relapse, several years had passed since PUVA therapy had been discontinued.

In summary, we report an extremely rare occurrence of MF superimposed on melanocytic nevi, and the lack of similar cases in the literature does not allow concrete explanations of this bizarre association. This report highlights the importance of clinical examination and appropriate employment of histology and immunohistochemistry, which together can lead to the right diagnosis of dermatological diseases.

\section{Disclosure Statement}

The authors declare that they have no conflict of interest.

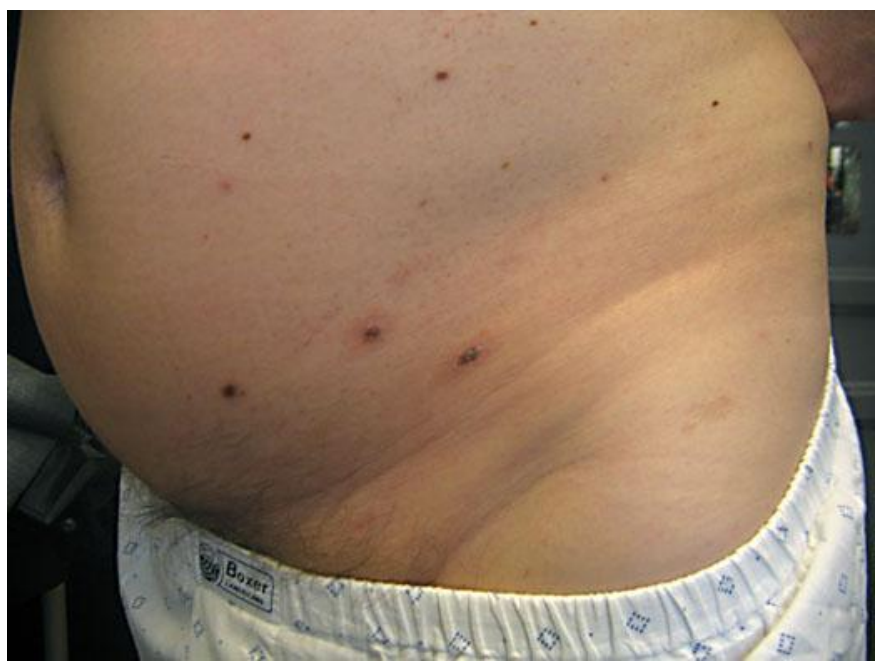

Fig. 1. Melanocytic nevi with epidermal scaling and an erythematous perinevic halo. 

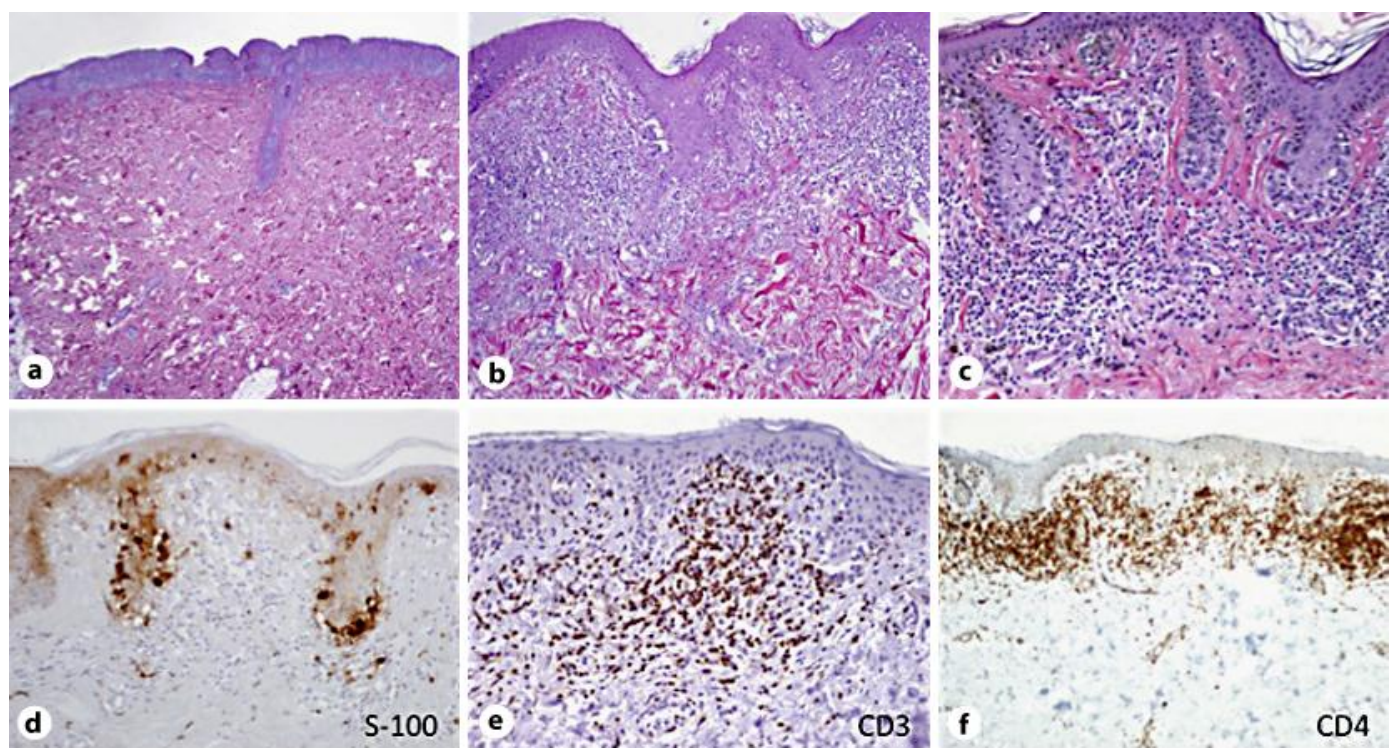

Fig. 2. a-c A dense infiltrate at the dermoepidermal junction composed by lymphocytes with hyperchromatic nuclei, exocytosis, and focal epidermotropism, together with junctional melanocytic nests. d Immunohistochemistry showing S-100 protein-positive staining in melanocytic nevus cells. e, $\mathbf{f}$ CD3+ and CD4+ lymphocytic infiltrate at the dermoepidermal junction and papillary dermis.

\section{References}

1 Galper SL, Smith BD, Wilson LD: Diagnosis and management of mycosis fungoides. Oncology 2010;24:491-501.

2 Keehn CA, Belongie IP, Shistik G, Fenske NA, Glass LF: The diagnosis, staging, and treatment options for mycosis fungoides. Cancer Control 2007;14:102-111.

-3 Kantor AF, Curtis RE, Vonderheid EC, Van Scott EJ, Fraumeni JF Jr: Risk of second malignancy after cutaneous T cell lymphoma. Cancer 1989;63:1612-1615.

4 Pielop JA, Brownell I, Duvic M: Mycosis fungoides associated with malignant melanoma and dysplastic nevus syndrome. Int J Dermatol 2003;42:116-122.

5 McNiff JM, Glusac EJ: Histologic features of melanocytic nevi seen in association with mycosis fungoides. J Cutan Pathol 2003;30:606-610.

-6 Olsen E, Vonderheid E, Pimpinelli N, Willemze R, Kim Y, Knobler R, Zackheim H, Duvic M, Estrach T, Lamberg S, Wood G, Dummer R, Ranki A, Burg G, Heald P, Pittelkow M, Bernengo MG, Sterry W, Laroche L, Trautinger F, Whittaker S, ISCL/EORTC: Revisions to the staging and classification of mycosis fungoides and Sezary syndrome: a proposal of the International Society for Cutaneous Lymphomas (ISCL) and the cutaneous lymphoma task force of the European Organization of Research and Treatment of Cancer (EORTC). Blood 2007;110:1713-1722.

7 Weinstock MA, Gardstein B: Twenty-year trends in the reported incidence of mycosis fungoides and associated mortality. Am J Public Health 1999;89:1240-1244.

$>8$ Boyd AS, Rapini RP: Cutaneous collision tumours. An analysis of 69 cases and review of the literature. Am J Dermatopathol 1994;16:253-257.

9 Nicholis DS, Mason GH: Halo dermatitis around a melanocytic naevus: Meyerson's naevus. Br J Derm 1988;118:125-129.

10 Shifer 0, Tchetchik R, Glazer 0, Metzker A: Halo dermatitis in children. Pediatr Dermatol 1992;9:275277.

11 Girardi M, Heald PW, Wilson LD: The pathogenesis of mycosis fungoides. New Engl J Med 2004;350:1978-1988. 
12 Tokura Y, Seo N, Yagi H, Takigawa M: Photoactivational cytokine-modulatory action of 8methoxypsoralen plus ultraviolet A in lymphocytes, monocytes and cutaneous T cell lymphoma cells. Ann NY Acad Sci 2001;941:185-193.

13 Tokura Y: Modulation of cytokine production by 8-methoxypsoralen and UVA. J Dermatol Sci 1999;19:114-122.

14 Larsson P, Andersson E, Johansson U, Öllinger K, Rosdahl I: Ultraviolet A and B affect human melanocytes and keratinocytes differently. A study of oxidative alterations and apoptosis. Exp Dermatol 2005;14:117-123.

15 Choi W, Miyamura Y, Wolber R, Smuda C, Reinhold W, Liu H, Kolbe L, Hearing VJ: Regulation of human skin pigmentation in situ by repetitive UV exposure: molecular characterization of responses to UVA and/or UVB. J Invest Dermatol 2010;130:1685-1696. 